\title{
Regulatory Small RNAs
}

\author{
V. A. Gvozdev \\ Institute of Molecular Genetics, Russian Academy of Sciences, pl. Kurchatova 2, \\ 123182 Moscow, Russia; E-mail: gvozdev@img.ras.ru
}

Received March 22, 2013

\begin{abstract}
Recent years have been marked by a burst of studies on the role of various RNAs in the regulation of gene expression. These regulatory effects act on the level of both chromatin in the nuclei and the cytoplasm during translation. The review papers of this issue are mainly dedicated to different types of small RNAs of 20-30 nucleotides. The small RNAs control diverse cellular functions including genome protection against transpositions of mobile elements of the genome.
\end{abstract}

DOI: $10.1134 / \mathrm{S} 0006297913060011$

Key words: microRNAs, siRNAs, piRNAs, heterochromatin, transposon

This issue of Biochemistry (Moscow) contains papers of Russian and foreign authors whose works contributed to the studies of the role of small RNAs (of 20-30 nucleotides) in the regulation of gene expression in various model organisms. Small RNAs also embrace the bestknown microRNAs, whose functions have been studied rather well. However, multiple functions of other types of small RNAs, siRNAs, and especially of piRNAs still need to be investigated. The manuscripts contained in this issue were written by authors who have contributed to knowledge about functioning of small RNAs. Under discussion is the role of small RNAs in the regulation of translation, formation of inactive "silent chromatin", and in defense of the cell against translocating mobile elements (transposons). Even this incomplete itemization of regulatory abilities of small RNAs shows their important role in cellular regulation believed to be a new "layer" of genetic information that up to recently somehow escaped the attention of researchers. Functioning of small RNAs involves their complementary interaction with cytoplasmic or nuclear transcripts by mechanism of the so-called RNA-interference (RNAi). Generally speaking, RNAi includes interactions of various small RNAs with the complementary sequences of cellular transcripts guided by proteins of the Argonaute family, which is evolutionary conserved but functionally extremely diverse. Pathways of these interactions and their biological effects are very variable. Some papers consider the role of piRNAs, the most diverse population of small RNA molecules responsible for very diverse functions as it has now become evident. The papers in this issue fail to address some problems concerning functions of small RNAs, in particular because of the worldwide burst-like character of studies in this field. Mechanisms of formation of "inactive heterochromatin" regions in eukaryotes are considered from different standpoints, and on reading separate papers the reader will understand why these words are put in quote marks. Certainly, many extremely important and interesting studies of biological effects of RNAi mechanism related, for instance, to cancer biology, neurobiology, or the struggle against infectious agents are not considered here. The manuscripts are written for an advanced and rather interested reader who, on comparing information from different papers on overlapping problems, will make some effort to imagine the state of this very rapidly developing field of molecular and cellular biology. 